\title{
Different expression of MIBI in primary site of non-Hodgkin lymphoma and its bone marrow deposits, a pilot study
}

This article was published in the following Dove Press journal:

Pathology and Laboratory Medicine International

27 February 2017

Number of times this article has been viewed

\author{
Jozef Malysz' \\ Juanita J Evans ${ }^{2}$ \\ Malcolm Acon-Laws ${ }^{3}$ \\ Michael G Bayerl' \\ Michael H Creer' \\ 'Department of Pathology, Penn State \\ Milton S Hershey Medical Center, \\ Hershey, PA, USA; ${ }^{2}$ Department \\ of Pathology, St. John Heath - \\ Providence, Southfield, MI, USA; \\ ${ }^{3}$ Laboratorio de Patologia Hospital \\ Cima, San Jose, Costa Rica
}

\begin{abstract}
Evaluation of mindbomb E3 ubiquitin protein ligase 1 (MIB1) (Ki67) proliferation index (PI) in B-cell non-Hodgkin lymphomas is increasingly a common addition to classification of lymphoma and staging procedures. Clinicians relay on PI as a surrogate marker of biologic activity; however, no established guidelines have been published whether PI at the primary site of the tumor gives the same answer as evaluation of tumor in staging marrow. In our study, dual immunohistochemical staining for MIB1 and CD20 was performed on tissue from primary site and bone marrow involved by B-cell non-Hodgkin lymphoma to compare PI for each individual patient. For all patients, MIB1 expression was higher at primary tumor site as compared to staging marrow. Additional analysis was performed to investigate the degree of difference depending on lymphoma morphology. Patients with large cell lymphoma at the primary site and large cell morphology in the marrow (LCL-L), those with large cell morphology at the primary site and small cell morphology in the marrow (LCL-S), and those with small cell morphology at the primary site and small cells in the marrow (SCL-S) were compared. As expected, LCL cases had a higher mean PI at the primary site when compared to SCL cases $(28.5 \%$ vs $2.8 \%$, $P=0.0001)$. In addition, the most significant difference between medullary and extramedullary PI was observed in cases with discordant morphology (LCL-S) $(21 \%$ vs $1.1 \%, P=0.009)$. Our results indicate that PI of lymphoma within the bone marrow should not be used as a surrogate prognostic indicator of lymphoma biology in its primary site.
\end{abstract}

Keywords: proliferation index, biologic behavior, prognosis

\section{Introduction}

Bone marrow (BM) biopsies are routinely performed for the staging of patients with most types of B-cell non-Hodgkin lymphomas (NHLs). On occasion, the hematologist/oncologist may request to determine the proliferation index (PI) of a lymphoma present within the staging marrow. Mindbomb E3 ubiquitin protein ligase 1 (MIB1) is a protein coding gene which has been regarded as a sensitive antibody useful in the determination of PI in paraffin-embedded tissues. ${ }^{1}$

Many previous studies have shown that an increased PI in certain types of B-cell NHLs is associated with a more aggressive disease course. ${ }^{2-8}$ These studies have been performed on diagnostic lymph nodes and other non-marrow tissues. We have encountered many requests for PI determination of the lymphoma on marrow specimens in cases where the marrow was the only diagnostic specimen. At other times, a diagnostic lymph node or other soft tissue-based lymphoma specimens are available. In either situation, the accuracy and significance of PI of lymphoma in staging marrow have not been clearly studied.
Correspondence: Jozef Malysz Department of Pathology, Penn State Milton S Hershey Medical Center, MC HI79, 500 University Drive, Hershey, PA I7033, USA

Tel $+|7| 753 \mid 1676$ (office)

Fax + I 717 53। 774I

Email jmalysz@hmc.psu.edu 
Our intent was to explore and characterize PI of B-cell NHLs in staging BMs and compare it to PI of the same patient at the primary, extramedullary tumor site in order to evaluate whether the MIB1 expression in BM involved by lymphoma can be used as an equivalent to that determined by staining of extramedullary primary tumor.

\section{Materials and methods}

In this retrospective study, the anatomic pathology database at Penn State Hershey Medical Center was searched for all patients diagnosed with B-cell NHL for whom a subsequent staging BM biopsies positive for involvement by lymphoma was also examined. A standard research protocol was reviewed and approved by Penn State Hershey Medical Center Institutional Review Board. Obtaining patient consent was deemed unnecessary due to the retrospective nature of this study. All patients meeting these criteria and evaluated within a 7-year period (2005-2012) at Hershey Medical Center were initially selected; however, patients with relapsed disease submitted for restaging and those treated prior to staging were excluded. Also, those for whom tissue samples either from primary or BM site was insufficient for additional staining were excluded. All slides were stained with hematoxylin and eosin and immunohistochemical stains were reviewed by two hematopathologists and hematopathology fellows for confirmation of the diagnosis and determination of percentage of MIB1-positive tumor cells. Commercially available immunohistochemical stains for MIB1 (permanent red chromogen with ethylenediaminetetraacetic acid [EDTA] antigen retrieval, 1:25 dilution, 30 minutes at room temperature) and CD20 (Diaminobenzidine [DAB] chromogen with EDTA antigen retrieval, 1:500 dilution, 30 minutes at room temperature) were obtained and used in a dual immunohistochemistry process that was performed on each specimen. Dual immunohistochemistry was performed using Dako's Envision G/2 Doublestain system on a Dako Autostainer Plus (Dako North America, Inc., CA, USA) following manufacturer's protocol. ${ }^{9,10}$ The PI for each site was determined by using MIB1 expression in 100 CD20positive lymphoma cells in a representative section. Clinical data, including patient demographics, sites of involvement, International Prognostic Index, and survival, were collected. The $P$-values were calculated using two-sample Student's $t$-test when comparing quantitative variables or by Chi-square test when comparing proportions, with significance set at a $P<0.05$.

In addition, a comparative study of MIB1 expression was performed using non-decalcified, formalin-fixed, paraffinembedded tissue and formalin-fixed, paraffin-embedded tissue after rapid decalcification (Decalcifier II [hydrochloric acid $<15 \%$; EDTA, disodium salt $<5 \%$ ], decalcification time 45 minutes), the same as used for decalcification of our BM samples. No significant difference of staining intensity, pattern, or percentage was identified in this comparison.

\section{Results}

Ample tissue at both sites of involvement to perform immunohistochemistry was present in 24 patients. The types of B-cell NHL included in this study, based on 2008 World Health Organization classification of the extramedullary site of involvement, were diffuse large B-cell lymphoma (eleven cases), follicular lymphoma (six cases), chronic lymphocytic leukemia/small lymphocytic lymphoma (four cases), extranodal marginal zone lymphoma (one case), and small B-cell lymphoma, not otherwise specified (two cases).

All cases were consolidated into three morphologic groups to increase statistical power of this analysis: large cell lymphoma at the extramedullary site with large cells in the BM (LCL-L), large cell lymphoma at the extramedullary site with discordant small cells in the BM (LCL-S), and small cell lymphoma at the extramedullary site with small cells in the BM (SCL-S) (refer to Table 1 for results).

In all cases, PI evaluated by MIB1 staining was higher at the extramedullary tumor site than that in the BM. Patients with LCL had a higher mean PI at the extramedullary site when compared to all SCL cases $(28.5 \%$ vs $2.8 \%, P=0.0001)$. The difference was statistically most significant for LCL-S ( $21 \%$ vs $1.1 \%, P=0.009)$, but the trend was observed in all subgroups. Figures 1-3 illustrate the common patterns of PI seen within the three morphologic subdivisions. Patients with large cell morphology at the extramedullary site had a lower mean survival than those with small cell morphology (18.1 vs 49.8 months, $P=0.002$ ). Similarly, patients with large cell morphology in the BM also had a lower mean survival than those with small cell morphology (9.3 vs 40.6 months,

Table I Mean and P-value of PI comparison at extramedullary tumor site versus BM sites in B-cell non-Hodgkin lymphomas

\begin{tabular}{lllll}
\hline Group & No of cases & $\begin{array}{l}\text { Extramedullary } \\
\text { site mean PI }\end{array}$ & $\begin{array}{l}\text { BM site } \\
\text { mean PI }\end{array}$ & P-value \\
\hline All cases & 24 & 14.6 & 3.6 & 0.009 \\
All LCL & $1 \mathrm{I}$ & 28.5 & 6.5 & 0.005 \\
LCL-L & 4 & 41.5 & 16 & 0.073 \\
LCL-S & 7 & 21 & 1.1 & 0.009 \\
All SCL-S & 13 & 2.8 & 1.1 & 0.057 \\
\hline
\end{tabular}

Note: The $P$-values were calculated using two-sample Student's $t$-test when comparing quantitative variables or by Chi-square test when comparing proportions, with significance set at a $P$-value $<0.05$.

Abbreviations: BM, bone marrow; LCL-L, large cell lymphoma at the primary site and large cell morphology in the marrow; LCL-S, large cell lymphoma at the primary site and small cell morphology in the marrow; PI, proliferation index; SCL-S, small cell morphology at the primary site and small cells in the marrow. 


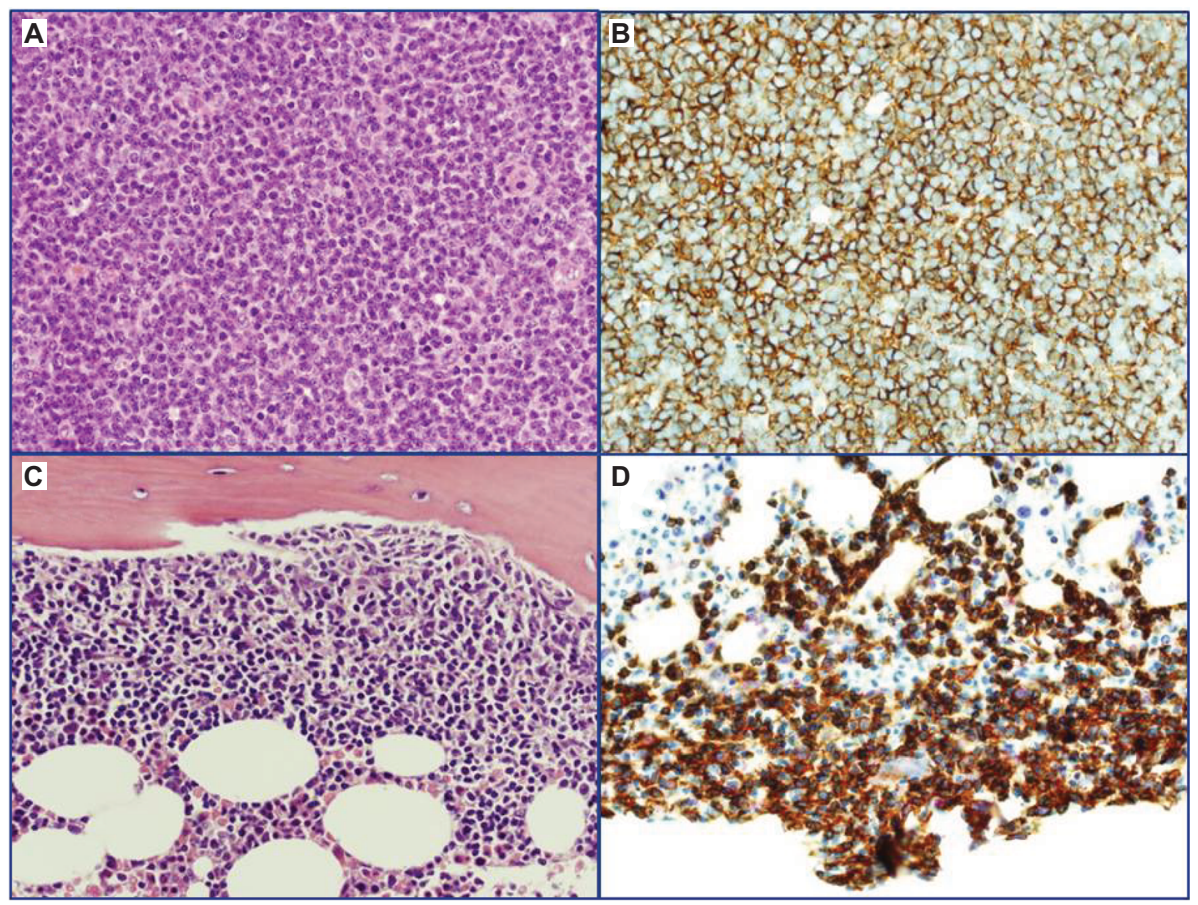

Figure I SCL-S.

Notes: (A) Low grade follicular lymphoma involving the lymph node (H\&E). (B) The same tissue as (A); dual stain: brown CD20 and red nuclear MIBI staining demonstrated low MIBI expression of $2 \%-3 \%$ (mean $2.8 \%$ ). (C) Follicular lymphoma of the same patient, involving the bone marrow (H\&E). (D) The same tissue as (C); dual stain demonstrated low MIBI expression of I\%-2\% (mean I.1\%); all images original, magnification $\times 500$.

Abbreviations: H\&E, hematoxylin and eosin; MIBI, mindbomb E3 ubiquitin protein ligase I; SCL-S, small cell morphology at the primary site and small cells in the marrow.

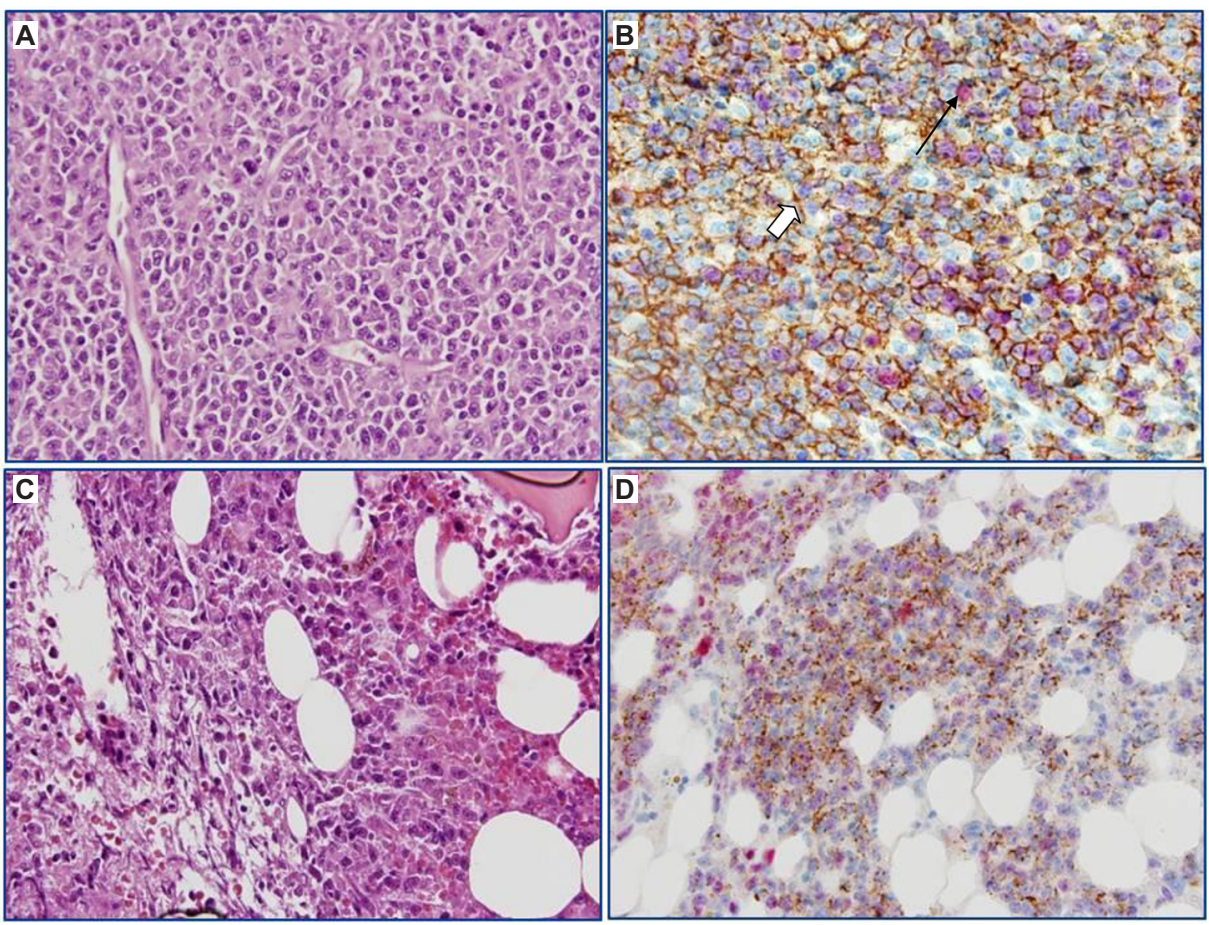

Figure 2 LCL-L.

Notes: (A) Diffuse large B-cell lymphoma (H\&E). (B) The same tissue as (A); dual stain demonstrated significantly higher MIBI expression (mean 4I.5\%); black arrow points to red nuclear staining of MIBI, white arrow points to brown cytoplasmic, and membranous staining of CD20. (C) Bone marrow of the same patient demonstrated predominance of large cells. (D) The same marrow with MIBI expression significantly lower than at its primary site (mean I6\%); all images original, magnification $\times 500$. Abbreviations: H\&E, hematoxylin and eosin; LCL-L, large cell lymphoma at the primary site and large cell morphology in the marrow; MIBI, mindbomb E3 ubiquitin protein ligase I. 


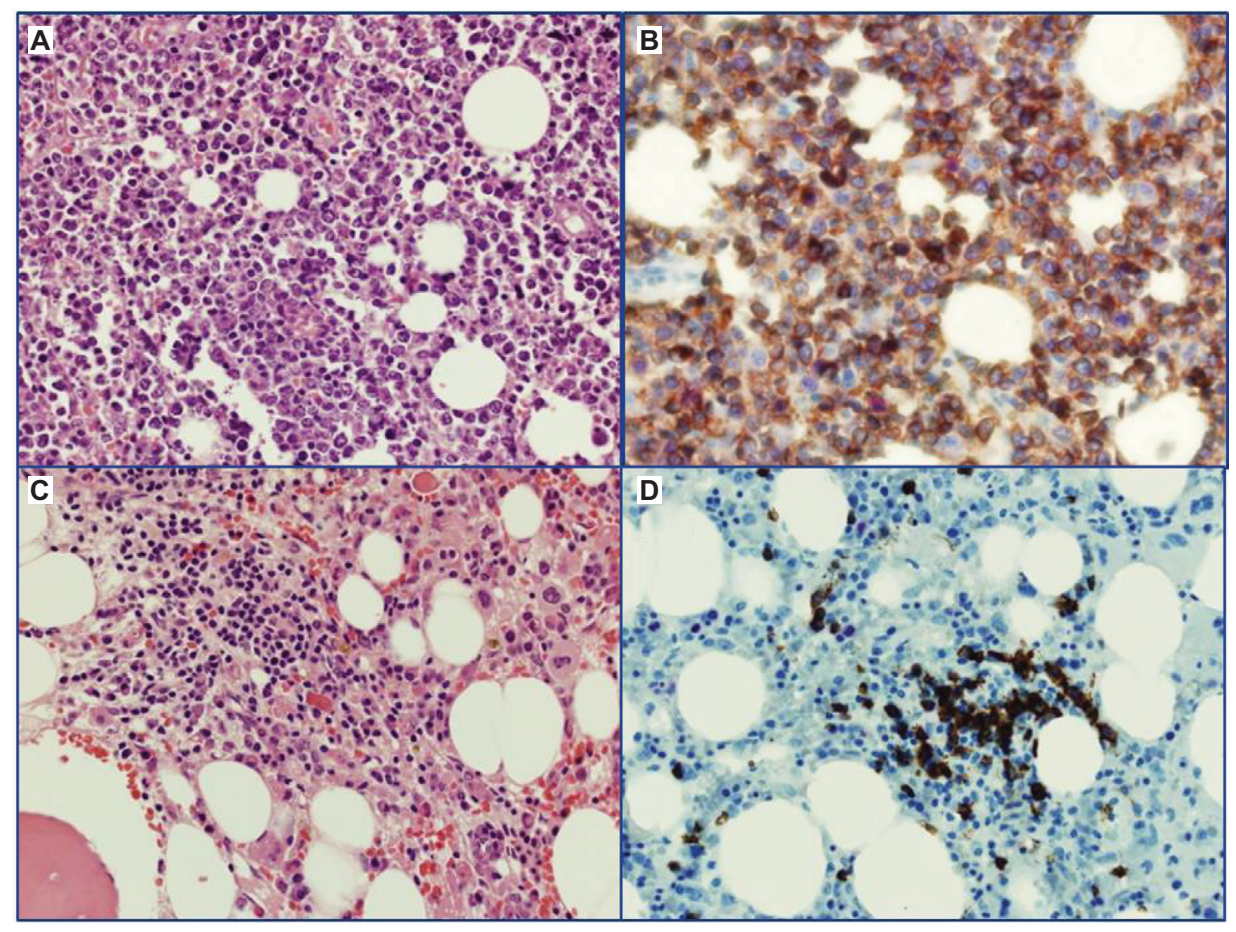

Figure 3 LCL-S

Notes: (A) Diffuse large B-cell lymphoma (H\&E). (B) CD20 and MIBI dual stain demonstrated high MIBI expression (mean $21 \%$ ). (C) Marrow of the same patient involved by lymphoma with predominantly small cells morphology (H\&E). (D) CD20 and MIBI dual stain demonstrated low MIBI expression (mean I.I\%); all images original, magnification $\times 500$.

Abbreviations: H\&E, hematoxylin and eosin; LCL-S, large cell lymphoma at the primary site and small cell morphology in the marrow; MIBI, mindbomb E3 ubiquitin protein ligase I.

$P=0.021)$. Higher mean PI at the extramedullary site was observed in all cases. When considering survival, patients surviving 1 year or less had a significantly higher PI at the primary site than those surviving longer than a year $(22.1 \%$ vs $10.8 \%, P=0.089)$. A similar trend was present with mean $\mathrm{PI}$ at the BM site in patients stratified by survival of 1 year or less versus greater than a year $(8.1 \%$ vs $1.3 \%, P=0.075)$.

\section{Discussion}

In our study, expression of MIB1 in lymphoma present in staging marrows was lower than the MIB1 expression at the extramedullary site in $100 \%$ of cases regardless of the morphology. The exact reason for this difference is unknown. It is possible that the BM has a different stromal and cytokine milieu than that of an extramedullary site, which may influence active proliferation of neoplastic cells. Discordance of morphology may also play a role. It is commonly accepted that discordant morphology between diffuse large B-cell lymphoma in its extramedullary location and its presence in the marrow can be due to diffuse large B-cell lymphoma arising from a preexisting low grade lymphoma or due to the presence of two clonally related or unrelated neoplasms. ${ }^{11}$ Our study documented higher PI between primary tumor site and that in the staging marrow in all cases studied.
We conclude similar to other investigators that morphology (large vs small cells) helps to predict biologic activity of the disease. PI in BM specimens consistently lower than that of a primary tumor site strongly suggests that BM specimens are not equivalent to biopsy of primary tumor site and should not be used interchangeably with primary biopsy site in the determination of tumor PI or disease biology. Thus, BM biopsy as a sole diagnostic tissue should not be regarded equivalent to the biopsy of primary tumor site for prognostic determination.

It is noteworthy that mean PI in the extramedullary sites of involvement is lower in our study than reported in literature. ${ }^{2,4,12}$ This finding is likely due to the use of a novel dual immunohistochemical staining technique optimized for staining in both BM and extramedullary sites. It must be recognized that native marrow cells can show up to $10 \%$ MIB1 expression in proliferating hematopoietic cells, ${ }^{13}$ and thus spuriously elevating PI in BM, which is not truly reflective of proliferation of neoplastic lymphocytes, but rather creates subjective impression of higher proliferation than that which can be attributed purely to lymphoma. Even though we did not specifically analyze the data with reference to degree of BM involvement or specific lymphoma subtype, dual immunohistochemistry for CD20 and MIB1 performed allowed more accurate assessment of PI of the neoplastic 
lymphocytes allowing separation of MIB1 staining in neoplastic B-cells from that of background hematopoietic cells. In fact, previous studies have observed wide interlaboratory and interobserver variability in MIB1 (Ki67) expression and suggested establishment of a laboratory-specific reference range for each immunohistochemical stain. ${ }^{2,8}$ Our technique significantly decreases the degree of bias attributable to this interference between proliferation of neoplastic cells and background marrow cells.

\section{Conclusion}

We conclude that for patients with BM involvement by B-cell NHL, the PI derived from BM should not be used as a surrogate prognostic indicator of PI of the lymphoma at its extramedullary site. Such inquiry may be treated as an additional source of clinically useful information, but if used inappropriately as an indicator of probable biologic behavior of lymphoma apart from the same determination at the primary tumor site, it may create a false impression of lower proliferative activity of the disease, which would be clinically misleading. We humbly recognize that our study is limited by the small number of studied cases and additional studies of this subject would be beneficial to further confirm or dispute our findings.

\section{Acknowledgment}

This study was funded by Penn State Milton S Hershey Department of Pathology Research Award Grant.

\section{Disclosure}

The authors report no conflicts of interest in this work.

\section{References}

1. Veronese SM, Maisano C, Scibilia J. Comparative prognostic value of Ki67 and MIB1proliferation indices in breast cancer. Anticancer Res. 1995;15(6B):2717-2722.

2. Broyde A, Boycov O, Strenov Y, et al. Role and prognostic significance of the ki-67 index in non-Hodgkin's lymphoma. Am J Hematol. 2009;84:338-343.

3. Hasselblom S, Ridell B, Sigurdardottir M, et al. Low rather than high $\mathrm{ki}-67$ protein expression is an adverse prognostic factor in diffuse large b-cell lymphoma. Leuk Lymphoma. 2008;49:1501-1509.

4. Yoon D, Choi D, Ahn H, et al. Ki-67 expression as a prognostic factor in diffuse large B-cell lymphoma patients treated with rituximab plus CHOP. Eur J Haematol. 2010;85:149-157.

5. Jovanovic M, Jakovic L, Bogdanovic A, et al. Poor outcome in patients with diffuse large b-cell lymphoma is associated with high percentage of bcl-2 and ki-67 positive tumor cells. Vojnosanit Pregl. 2009;66:738-743.

6. Gaudio F, Giordano A, Perrone T, et al. High ki-67 index and bulky disease remain significant adverse prognostic factors in patients with diffuse large $\mathrm{b}$ cell lymphoma before and after the introduction of rituximab. Acta Haematol. 2011;126:44-51.

7. Salles G, de Jong D, Xie W, et al. Prognostic significance of immunohistochemical biomarkers in diffuse large b-cell lymphoma: a study from the Lunenburg Lymphoma Biomarker Consortium. Blood. 2011;117:7070-7078.

8. Ali A, Morgen E, Geddie W, et al. Classifying b-cell non-Hodgkin lymphoma by using MIB-1 proliferative index in fine-needle aspirates. Cancer Cytopathol. 2010;118:166-172.

9. EnVision ${ }^{\mathrm{TM}} \mathrm{G} \mid 2$ Doublestain System, Rabbit/Mouse (DAB+/Permanent Red) Code K5361 2nd edition/. Available from: http://www.dako.com/ us/ar49/p235046/prod_products.htm

10. Monoclonal Mouse Anti-Human Ki-67 Antigen Clone MIB-1 Code M7240 (specification sheet 104980-004) Dako Agilent Technology Company. Available from: http://www.dako.com/us/ar38/p104960/ prod_products.htm. Accessed August 22, 2016.

11. Kremer M, Spitzer M, Mandl-Weber S, et al. Discordant bone marrow involvement in diffuse large b-cell lymphoma: comparative molecular analysis reveals a heterogeneous group of disorders. Lab Invest. 2003;83:107-114.

12. Kalogeraki A, Tzardi M, Panagiotides I, et al. MIB1 (Ki-67) expression in non-Hodgkin lymphomas. Anticancer Res. 1997;17:487-491.

13. Shin S, Sheibani K, Kezirian J, et al. Immunoarchitecture of normal bonemarrow: a study of frozen and fixed tissue sections. Hum Pathol. 1992;23:686-694.
Pathology and Laboratory Medicine International

\section{Publish your work in this journal}

Pathology and Laboratory Medicine International is a peer-reviewed, open access journal focusing on innovative basic research and translational research related to pathology or human disease. The journal includes original research, updates, case reports, reviews and commentaries on current controversies. The manuscript management system is completely

\section{Dovepress}

online and includes a very quick and fair peer-review system. Visit http://www.dovepress.com/testimonials.php to read real quotes from published authors. 